\title{
Refinements in the use of equivalent latitude for assimilating sporadic inhomogeneous stratospheric tracer observations, 1: Detecting transport of Pinatubo aerosol across a strong vortex edge
}

\author{
P. Good ${ }^{1}$ and J. Pyle ${ }^{2}$ \\ ${ }^{1}$ Institute of Environmental Research and Sustainable Development, National Observatory of Athens, Greece \\ ${ }^{2}$ Centre for Atmospheric Science, Cambridge, UK
}

Received: 2 December 2003 - Published in Atmos. Chem. Phys. Discuss.: 29 January 2004

Revised: 31 Auguat 2004 - Accepted: 2 September 2004 - Published: 13 September 2004

\begin{abstract}
The use of PV equivalent latitude for assimilating stratospheric tracer observations is discussed - with particular regard to the errors in the equivalent latitude coordinate, and to the assimilation of sparse data. Some example measurements are assimilated: they sample the stratosphere sporadically and inhomogeneously. The aim was to obtain precise information about the isentropic tracer distribution and evolution as a function of equivalent latitude. Precision is important, if transport across barriers like the vortex edge are to be detected directly. The main challenges addressed are the errors in modelled equivalent latitude, and the non-ideal observational sampling. The methods presented allow first some assessment of equivalent latitude errors and a picture of how good or poor the observational coverage is. This information determines choices in the approach for estimating as precisely as possible the true equivalent latitude distribution of the tracer, in periods of good and poor observational coverage. This is in practice an optimisation process, since better understanding of the equivalent latitude distribution of the tracer feeds back into a clearer picture of the errors in the modelled equivalent latitude coordinate. Error estimates constrain the reliability of using equivalent latitude to make statements like "this observation samples air poleward of the vortex edge" or that of more general model-measurement comparisons. The approach is demonstrated for groundbased lidar soundings of the Mount Pinatubo aerosol cloud, focusing on the 1991-92 arctic vortex edge between 475$520 \mathrm{~K}$. Equivalent latitude is estimated at the observation times and locations from Eulerian model tracers initialised with PV and forced by UK Meteorological Office analyses. With the model formulation chosen, it is shown that tracer transport of a few days resulted in an error distribution that
\end{abstract}

Correspondence to: P. Good

(pgood@meteo.noa.gr) was much closer to Gaussian form, although the mean error was not significantly affected. The analysis of the observations revealed a small amount of irreversible transport of aerosol across the vortex edge during late January 1992, coincident with a strongly disturbed vortex.

\section{Introduction}

The environmental effects of anthropogenic emissions into the atmosphere are strongly dependent on the transport of chemical constituents. For example, a crucial factor controlling the depletion of stratospheric ozone is the strength of the winter polar vortex (e.g. Chipperfield and Jones, 1999; Millard et al., 2002). Long-lived constituents act as dye-like tracers of transport. Tracer transport timescales vary greatly, e.g. with rapid transport parallel to the vortex but very slow transport across it. The vortex distorts continuously, exhibiting very fine scale features (e.g. Plumb et al., 1994) and so its position and size cannot be precisely known. Ground-based tracer observations tend to be sporadic and inhomogeneous, whereas current satellite data is of relatively low spatial resolution. Measuring the slow irreversible transport of tracers across barriers like the vortex edge, by comparing sets of measurements with themselves and with model results, then, is not straightforward. This kind of irreversible transport has been inferred for other periods indirectly using interrelationships between long-lived tracers. Tracer-tracer interrelationships are a very powerful tool (Plumb and Ko, 1992), but can be misinterpreted (see e.g. Plumb et al., 2000).

In the stratosphere in particular, tracer analysis may be simplified by using carefully chosen coordinate systems. Meridional coordinates based on the area enclosed by contours of potential vorticity (PV) or other tracers have been 
used (e.g. Butchart and Remsberg, 1986; Nakamura, 1996), since the area enclosed by a tracer contour on an isentropic surface evolves much more slowly than reversible deformations in its shape. Butchart and Remsberg (1986) defined the "equivalent latitude" for PV, but it is expressed more generally as $\phi_{e}=\sin ^{-1}(1-2 A(\chi))$, where $A(\chi)$ is the area enclosed by a tracer contour of value $\chi$ on a normalised sphere. Nakamura (1996) showed that when a contour area coordinate is used, two dimensional isentropic transport behaves as a diffusion. It is reasonable to use equivalent latitude calculated from a single long lived tracer as an approximate meridional coordinate, because long lived tracers tend to show relatively tight correlations, due to the rapid local mixing processes (Plumb and Ko, 1992). Since this coordinate system is based partly on numerical model simulations, understanding and minimising errors in equivalent latitude estimates is crucially important for precise diagnosis of tracer transport from observations of the real atmosphere. Throughout this paper, the term "true equivalent latitude" is used to indicate equivalent latitude that would be derived, were it possible, from the observed tracer in real atmosphere.

$\mathrm{PV}$ is convenient but not ideal for calculating equivalent latitude: PV is conserved in the lower stratosphere over timescales only of the order of days - so does not necessarily correlate sufficiently well with long lived tracers; and its diagnosis from grid-point meteorological fields tends to incur errors.

Numerical passive tracer transport of PV can improve correlation with observed long lived tracers, and hence can reduce errors in the associated equivalent latitude. Lagrangian transport has been demonstrated for generating high resolution fields from PV (e.g. O'Neill et al., 1994; Fairlie et al., 1997; Flentje et al., 2000; Dragani et al., 2002), global model tracer fields (e.g. Methven and Hoskins, 1999; Evans et al., 2000; Methven et al., 2001, 2003) and gridded satellite data (e.g. Sutton et al., 1994; Manney et al., 1998). During high resolution model tracer transport, chaotic stirring of the tracer shears errors to small spatial scales without removing the errors, while inaccuracies in the model transport will add further errors. Another effect of tracer transport is that local mixing can smooth errors: this is the mechanism which drives the tightening of tracer-tracer correlations (e.g. Plumb and Ko, 1992). Pure Lagrangian transport will offer only the first potential benefit, while the benefit of Eulerian transport will be resolution and model dependent. For Lagrangian models, spatial averaging or interpolation of initialisation or output (e.g. Manney et al., 1998) can mimic local mixing, removing those errors which have been sheared to sufficiently small scale. Allen and Nakamura (2003) demonstrate some success with equivalent latitude fields derived from Eulerian model passive tracers.

In order to evaluate the chosen coordinate system, it is important to test it against high resolution observations, although this is by no means straightforward. Flentje et al. (2000) compared the spatial positions of modelled and ob- served tracer filaments and found significant benefits from (Lagrangian) contour advection of PV. Their results reproduced more than $90 \%$ of observed ozone and aerosol filaments to within $1^{\circ}$. Fairlie et al. (1997) tested correlations between observed $\mathrm{N}_{2} \mathrm{O}$ and predictions based on PV tracers: they found 5-day Lagrangian trajectory transport of PV in general reduced the ability to predict observed airborne $\mathrm{N}_{2} \mathrm{O}$ mixing ratios. These results are not necessarily inconsistent: a model might reproduce the position of a filament well, but large spatial gradients may translate to large errors in the modelled tracer value at any given point. That is to say, Lagrangian advection might reduce the spatial scale of tracer errors, but the magnitude of these errors might actually increase. Good et al. (2003) investigated the amount of small scale tracer structure generated by Lagrangian advection of low resolution Eulerian fields, finding mix-down times which allowed the advection to reproduce the amount of small scale structure found in high resolution aircraft data.

The analysis presented below is new because it evaluates errors in equivalent latitude - rather than tracer mixing ratio or filament position. It further makes different assumptions about the relationship between the modelled and observed tracers, and uses a very large number of relatively high resolution observations. Equivalent latitude is fixed relative to contour area and so makes a useful general coordinate for assessing transport; PV or other tracers evolve in different ways. Also, equivalent latitude error results are straightforward to interpret and intercompare.

Understanding the error in equivalent latitude helps direct its use in assimilating atmospheric observations. For a single measurement, the confidence in statements such as "this measurement corresponds to air inside the vortex edge" may be addressed. With a large number of observations, methods may be chosen for combining them to filter out noise. Straightforward averaging may not always provide the best results. Some possibilities are demonstrated below.

The basic tool used is the probability distribution function (PDF), specifically the probability $\mathrm{P}\left(v>v_{i}\right)$, where $v$ is a tracer mixing ratio, and $v_{i}$ a chosen threshold value of that tracer mixing ratio. These PDFs are calculated as a function of diagnosed equivalent latitude. Sparling (2000) studied PDFs of stratospheric tracers, but expressed them as functions of latitude instead of equivalent latitude. The use of PDFs calculated as a function of equivalent latitude is a new and important part of the current study, since it filters out many of the short term reversible atmospheric motions, leaving a clearer picture of the significant tracer distribution.

The observations studied below track the stratospheric sulphate aerosol cloud resulting from the June 1992 Mount Pinatubo eruption. See Russell et al. (1996) and references therein for detailed discussion of the ensemble of Pinatubo aerosol observations. These show a relatively stable reservoir of volcanic aerosol in northern midlatitudes by early 1992, and a rather clean arctic lower stratospheric vortex. A significant deformation of the vortex during late January 1992 
Table 1. References for the EASOE lidar data.

\begin{tabular}{llll}
\hline Site & Position & Citation & $\begin{array}{l}\text { Author of } \\
\text { CD-ROM data }\end{array}$ \\
\hline Haute-Provence & $44.5^{\circ} \mathrm{N}, 5.5^{\circ} \mathrm{E}$ & Chazette et al. (1995) & S. Godin \\
Aberystwyth & $52.4^{\circ} \mathrm{N}, 355.9^{\circ} \mathrm{E}$ & Vaughan et al. (1994) & G. Vaughan \\
Sodankyla & $67.4^{\circ} \mathrm{N}, 26.6^{\circ} \mathrm{E}$ & Stein et al. (1994) & L. Stefanutti \\
Andoya & $69.3^{\circ} \mathrm{N}, 16.0^{\circ} \mathrm{E}$ & Schafer et al. (1994) & K. H. Fricke \\
Thule & $76.5^{\circ} \mathrm{N}, 291.2^{\circ} \mathrm{E}$ & Digirolamo et al. (1994) & G. Fiocco \\
Ny-Ålesund & $78.9^{\circ} \mathrm{N}, 11.9^{\circ} \mathrm{E}$ & Beyerle and Neuber (1994) & R. Neuber \\
\hline
\end{tabular}

has been detected in model and measurements (Plumb et al., 1994), but clear direct evidence of irreversible tracer transport across the vortex edge has not previously been shown. Plots of observations against diagnosed equivalent latitude are insufficient, since it is always possible that apparent poleward transport is due to errors in the equivalent latitude coordinate. As well as testing a set of new analysis methods, the aim of the study below was to discover whether real irreversible transport of Pinatubo aerosol into the arctic vortex actually occurred in the potential temperature slice 475$520 \mathrm{~K}$ during early 1992. Good and Pyle (2004) provide further analysis, showing the variation with potential temperature of the irreversible transport of Pinatubo aerosol to very high northern latitudes during early 1992. Good and Pyle (2004) show that poleward transport increased below $475 \mathrm{~K}$ and was negligible above $520 \mathrm{~K}$, so the interval $475-520 \mathrm{~K}$ is a kind of transition region.

After presentation of model and measurements, the error characteristics of the diagnosed equivalent latitude coordinate are investigated. The effect of model transport on these errors is highlighted. Building on this, two new methods of estimating the true equivalent latitude distribution of the observed tracer are described. "True equivalent latitude" is used to indicate equivalent latitude that would be derived, were it possible, from the observed tracer in real atmosphere. These two methods are used to study transport of the observed tracer in the region of the vortex edge in the potential temperature slice 475-520 K during early 1992.

\section{Model and measurements}

\subsection{Equivalent latitude tracers}

Equivalent latitude fields are here estimated from PV tracers, transported by the SLIMCAT isentropic Eulerian 3d model, which has previously been used successfully for studies of the stratospheric photochemistry and transport (see Chipperfield, 1999). United Kingdom Meteorological Office analyses (described by Swinbank and O'Neill, 1994) were used to calculate the initial PV fields, and to force the model transport. SLIMCAT uses the Prather advection scheme (Prather,
1986). For these experiments, diabatic heating was set to zero, and horizontal transport calculated on 2 isentropic levels, at $475 \mathrm{~K}$ and $520 \mathrm{~K}$. The horizontal resolution was $5.6^{\circ}$ in longitude and $2.5^{\circ}$ in latitude, chosen to give the grid boxes approximately unit aspect ratio at typical polar vortex edge latitudes.

The experiment was configured, such that for any modelled time, five PV tracers were available. Each PV tracer was initialised with diagnosed PV at $00 \mathrm{~h}$ GMT on a given date and then transported for up to five days. The only difference between the five tracers was their date of initialisation, and hence the length of time for which they had been transported. Equivalent latitude fields were then calculated from each PV tracer; these fields are denoted $\phi_{e(1)}, \phi_{e(2)}$, $\phi_{e(3)}, \phi_{e(4)}, \phi_{e(5)} . \phi_{e(n)}$ refers to equivalent latitude calculated from a PV tracer which was initialised between $n$ and $n-1$ days earlier. For example, $\phi_{e(5)}$ derived at $17 \mathrm{~h}$ GMT for 28 January is based on a PV tracer which was initialised at $00 \mathrm{~h}$ GMT on 24 January. These equivalent latitude fields were interpolated linearly to each measurement location and time. Unless otherwise stated, $\phi_{e(5)}$ is used, since this is found below to perform best in assimilating the observations. A maximum integration time of five days was used in order to keep the computational burden down. Allen and Nakamura (2003) demonstrate a new method for calculating a PV-like tracer which may be smoother at high latitude, and could be used in future studies. The model resolution and interpolation method was chosen to be consistent with the configurations of SLIMCAT typically in use for photochemical transport studies at the time of writing.

\subsection{Observed data}

This analysis uses ground-based $532 \mathrm{~nm}$ aerosol lidar backscatter observations taken at six sites at mid- and high northern latitudes during the EASOE campaign of winter 1991-92. The measurements are available on the EASOE CD-ROM. See Table 1 for locations and references describing the measurement data. The vertical resolution is mostly of $200 \mathrm{~m}$ or less, or about $4 \mathrm{~K}$ in the lower stratosphere. This is equivalent (Haynes and Anglade, 1997) to about $50 \mathrm{~km}$ in the horizontal. 
a)

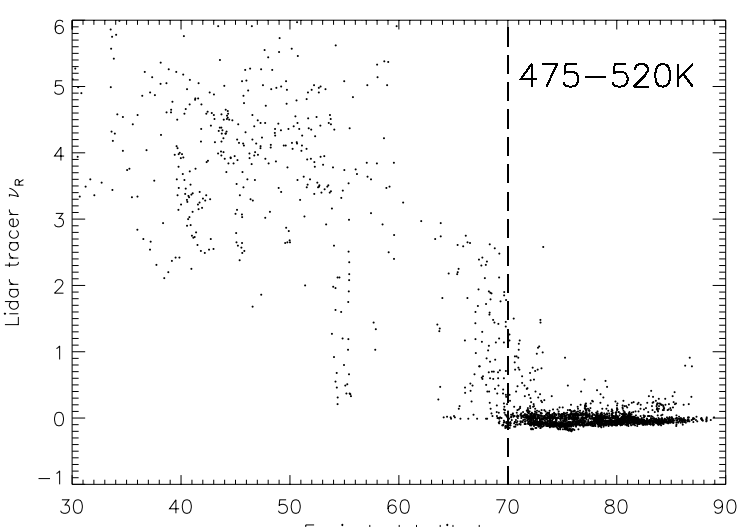

b)

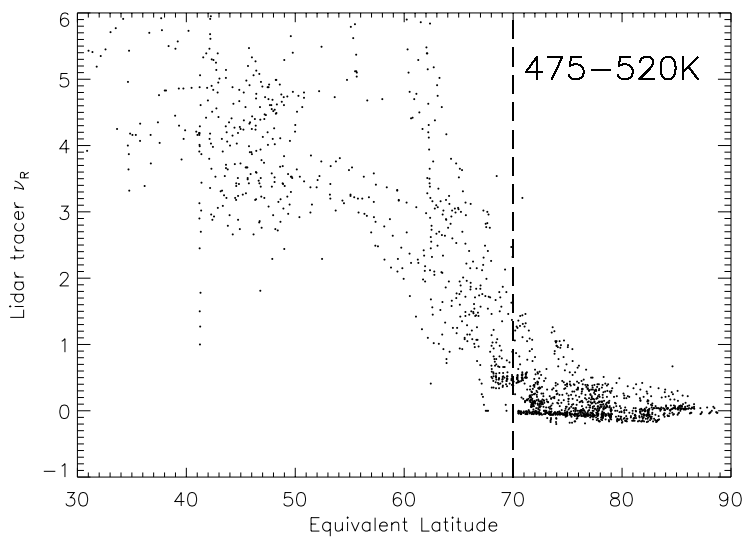

Fig. 1. Observed $v_{R}$ versus $\phi_{e(5)}, 475-520 \mathrm{~K}$. (a) Days $0-40$ of 1992; (b) days 41-80.

\subsubsection{Aerosol backscatter as a suitable passive tracer during} early 1992

From the aerosol backscatter ratio (R), the best estimate of a conserved tracer mixing ratio ( $v_{R}$, or "lidar tracer") is given by $v_{R}=\mathrm{R}-1$ (see Bohren and Huffman, 1981) and the references in Table 1].

Brock et al. (1993) and Jager et al. (1995) used observed aerosol size distributions to show that for the case of $532 \mathrm{~nm}$, lidar backscatter ratio is a good predictor (to within 5\%) of aerosol mass mixing ratio during late 1991 through early 1992.

The only sulphate aerosol microphysical processes significantly modifying mass mixing ratio during early 1992 (Russell et al., 1993) were gravitational sedimentation, water vapour uptake and PSC formation: these could affect conservation of $v_{R}$ and hence its usefulness as a passive tracer. Sedimentation rates may be estimated (G. Brasseur and Tyndall, 1999) using observed size distributions (e.g. Deshler et al., 1993; Deshler, 1994). The effects of water vapour uptake on the lidar observations were estimated using standard thermodynamic data (Hanson et al., 1994; Steele and Hamill, 1981) and Mie backscatter calculations (see Bohren and Huffman,
1981). This effect is negligible for these specific observations, since the regions of low temperatures either had very low volcanic aerosol content or were not sampled by the observations. The northern winter 1991-92 saw only a short period of low stratospheric temperatures between late December 1991 and mid January 1992; too warm for PSC II (e.g. Farman et al., 1994). The observation of PSCs of any type during early 1992 was relatively infrequent, and PSC Ia are identified by high depolarisation and return relatively low backscatter signals (Browell et al., 1990). Plots of backscatter ratio versus analysed temperature for the early 1992 lidar data show PSC Ib only over Andoya on 9 January. This PSC was also observed by the ISAMS satellite instrument (Massie et al., 1997). These observations were removed before analysis.

The random error in the lidar data was estimated empirically at about $10-20 \%$ in R. This estimate used the scatter of values measured within the midlatitude surf-zone, with the mean vertical gradient removed. Horizontal tracer gradients are small within the surf-zone, so measurement error dominates over equivalent latitude error. This is consistent with reported theoretical error estimates (references in Table 1).

\subsection{Data selection}

Data is analysed for the potential temperature interval 475$520 \mathrm{~K}$ because this interval shows a large ratio in observed $v_{R}$ between midlatitudes and vortex centre - the vortex edge step is large compared to measurement error (Fig. 1). Most attention is on the vortex edge, where the scatter of points is large compared to measurement error: here the error in equivalent latitude is dominant. The vortex edge was relatively poorly sampled during January, but the vortex core was very well sampled.

\section{Error in vortex-edge equivalent latitude with respect to backscatter observations}

The statistics of the relationship between observed $v_{R}$ and diagnosed equivalent latitude were investigated first. The aim was to understand the errors in the equivalent latitude coordinate in the region of the vortex edge, in order to direct useful ways of filtering these errors out and hence obtain a clear picture of the distribution of the observed tracer in equivalent latitude. In this paper we are interested in the problem of detecting transport of a tracer across a strong vortex edge. For this purpose, the error in equivalent latitude needs to be characterised only in the region of the vortex edge.

Figure 2 shows estimates of the probability distribution functions (PDFs) $P\left(v_{R}>v_{R(i)}\right)$, with $v_{R(i)}$ being a constant for each curve. This is a cumulative PDF, and if there were no errors in either diagnosed equivalent latitude or in the measurements, these curves would all be step functions. PDFs are calculated for consecutive $2.5^{\circ}$ equivalent latitude bins. 


\section{Number of measurements per bin}

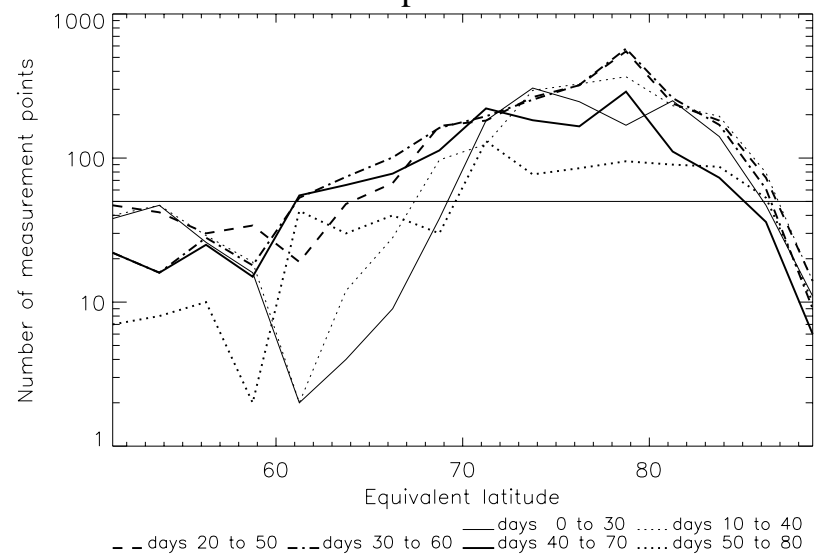

Probability distributions for $\nu_{R}>\nu_{R(i)}$
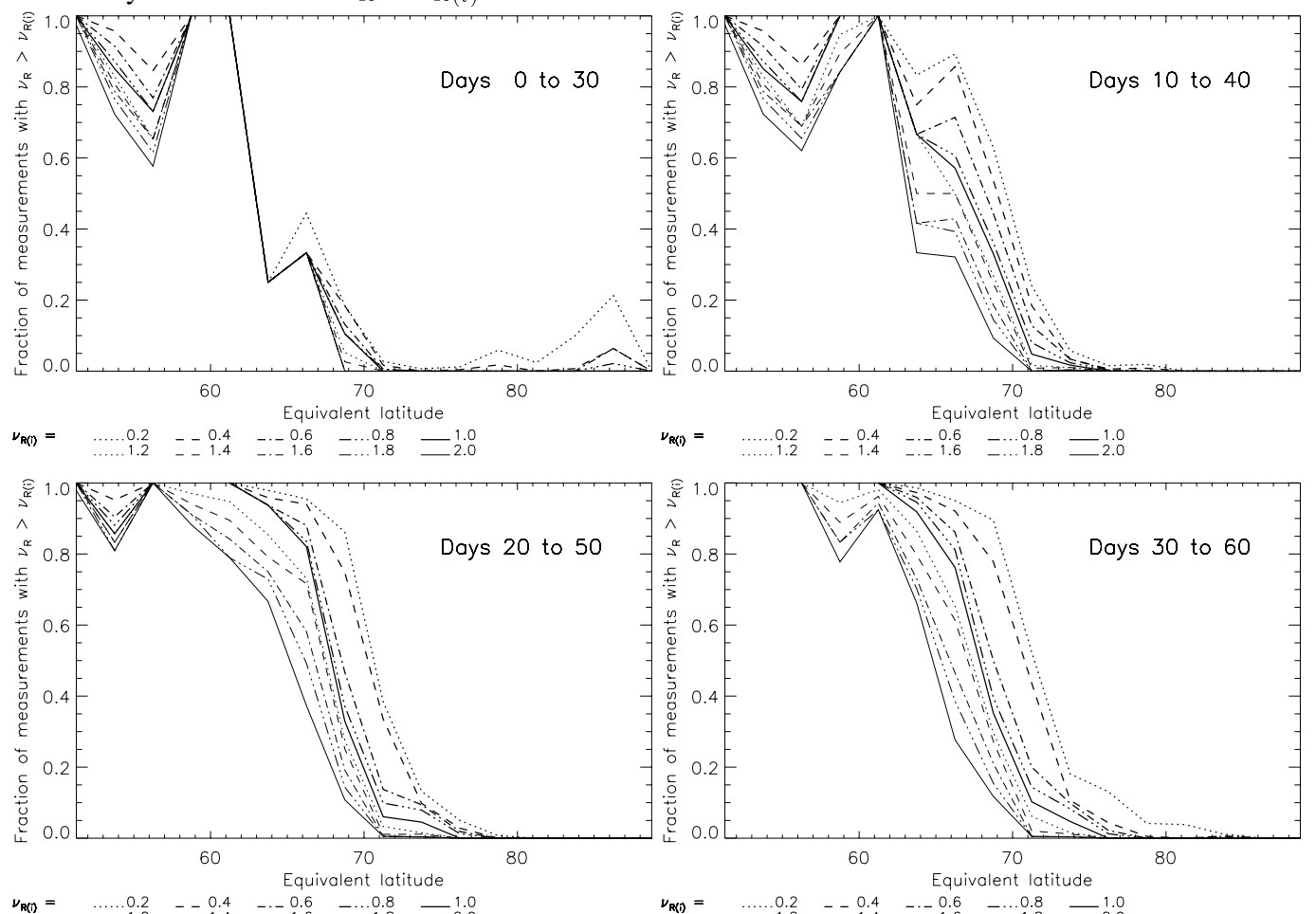

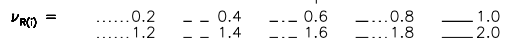
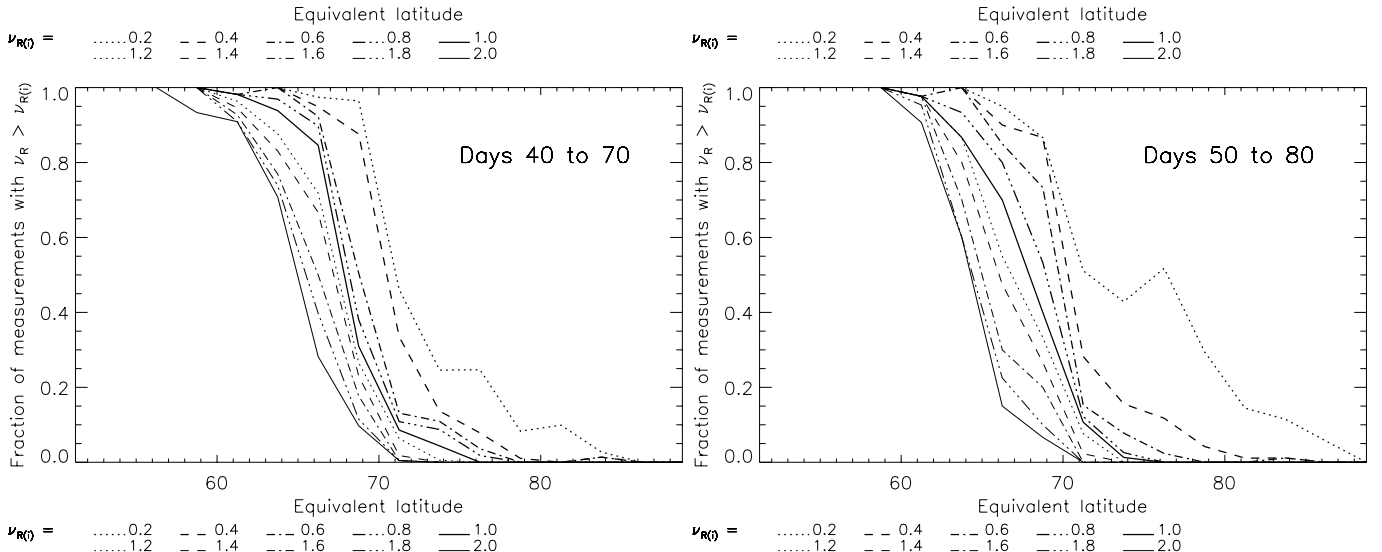

Fig. 2. Top: number of measurements in each $2.5^{\circ}$ equivalent latitude bin. The horizontal line $\mathrm{N}=50$ marks an approximate, empirical minimum threshold for reliable probability distributions (see text). Main: probability distributions for $\left(v_{R}>v_{R(i)}\right)$, for selected values of $v_{R(i)}$ from 0.2 to 2.0. $v_{R(i)}$ decreases with $\phi_{e}$. Dates are in Julian days of 1992. 


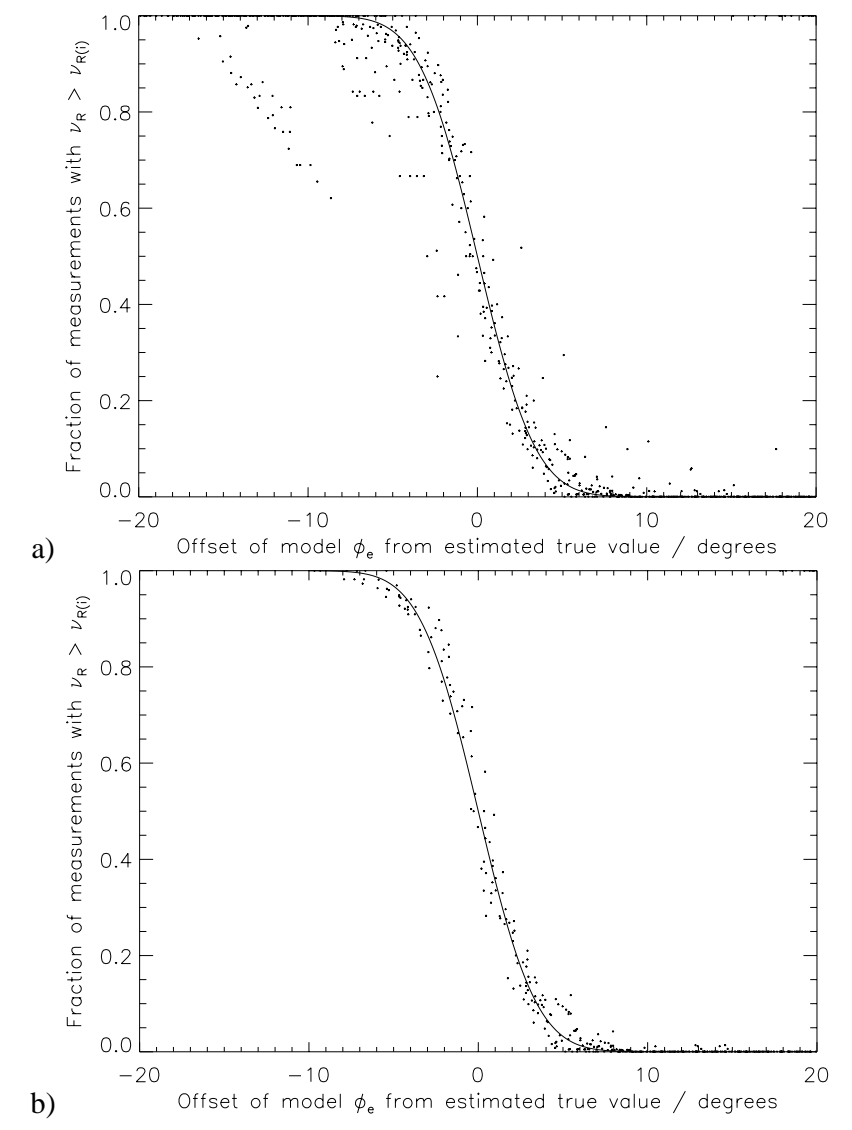

Fig. 3. PDFs from Fig. 2, but with the best estimate of $\phi_{e(t)}$ removed. (a) all PDFs; (b) bins with at least 50 measurements, and $v_{R(i)} \geq 0.4$.

Each panel summarises the data for a time bin of width 30 days; the time bins are spaced at 10 day intervals, so they overlap. Dates are given throughout as days of the year 1992 ("day 0" being 1 January 1992). The plots cover the first 80 days of 1992 (through $20 \mathrm{March}$ ). The top panel shows the total number of measurements in each equivalent latitude bin, each curve corresponding to a different time bin. The PDFs are calculated for selected values of $v_{R(i)}$ from 0.2 to 2.0. This selects only those measurements which are clearly part of the steep tracer gradient associated with the vortex edge (see Fig. 1): here the measurement error is unimportant for the correlation between $v_{R}$ and equivalent latitude. The use of these PDFs below assumes that in the absence of any errors, observed $v_{R}$ would be locally monotonic in equivalent latitude ("locally" means over the scale of equivalent latitude error): i.e. that the sign of the gradient of $v_{R}$ with equivalent latitude is known.

The PDFs are introduced best by the plot for Julian days 20-50. These curves all follow a reasonably similar function of $\phi_{e}$. This general shape is expected if there is an approximately Gaussian error in model $\phi_{e}$ within the vortex edge.
In fact, this shape is repeated to some extent for all time windows. Obvious exceptions occur mostly where the number of measurements in a bin is less than about 50. These may be counted as noise due to bad statistics. In particular, there is a low measurement coverage at $\phi_{e}<70^{\circ}$ prior to Julian day 20 and to a lesser extent after day 50 . The small spike at very high $\phi_{e}$ in the plot for Julian days 0-30 is very likely to be due to PSCs, since the analysed temperatures corresponding to these observations are all less than $195 \mathrm{~K}$ and all but one of these observations are at the same location and within a day of the PSC Ib observations identified in Sect. 2.3. The PDFs for $v_{R(i)}=0.2$ after day 50 show elevated levels at high $\phi_{e}$. This seems to show a small amount of transport of aerosol into the vortex after day 50 .

\subsection{A quantified error estimate for the vortex edge region}

Since the PDFs presented in the previous section have a shape similar to that expected from Gaussian error statistics, then it is possible to estimate the magnitude of the error by fitting the appropriate curve. This error magnitude indicates the quality of $\phi_{e}$ - the quality as a coordinate for assimilating the Pinatubo aerosol lidar data. It predicts the random error in an equivalent latitude value $\phi_{e}$ diagnosed for a given observed lidar tracer value $v_{R(i)}$, in the vortex edge region.

This coordinate random error arises both from inadequacies in the $\phi_{e}$ diagnosis and from specifics of the observed lidar tracer. Specifics of the lidar tracer include measurement random error, aerosol microphysics - in particular, sedimentation - and the fact that two different passive tracers will show tight, but not perfect correlations (e.g. Plumb and Ko, 1992). The remaining coordinate random error is due to the error in $\phi_{e}$ diagnosis, of crucial importance to the use of $\phi_{e}$ in assimilating observed data.

The PDFs in Fig. 2 have rather similar shapes, except where the number of measurements is low. This common shape is approximately that which would be expected from a Gaussian error in $\phi_{e}$. In order to estimate the magnitude of this errror, Gaussian statistics were assumed and the appropriate curve fitted independently to each PDF in Fig. 2, with the curve mean and width as fit parameters. For each fit, the squared deviation in probability (the ordinate) was minimised, weighted by the reciprocal of the square of the number of measurements in each bin. Thus, bins with few measurements affect the curve fit only weakly. This system of weighting was found empirically to give reasonable fits, without being influenced by the noise from bins with few measurements. The average of the fitted widths was $2.7^{\circ}$. Errors in both measurements and equivalent latitude contribute to this width, but the measurement error magnitude has been estimated already. Once the effect of measurement random error is subtracted, our best estimate of $\phi_{e}$ coordinate error is $2.6 \pm 0.25^{\circ}$.

Figure 3 is used to demonstrate that the estimated Gaussian error is representative of the vortex edge data. The solid 

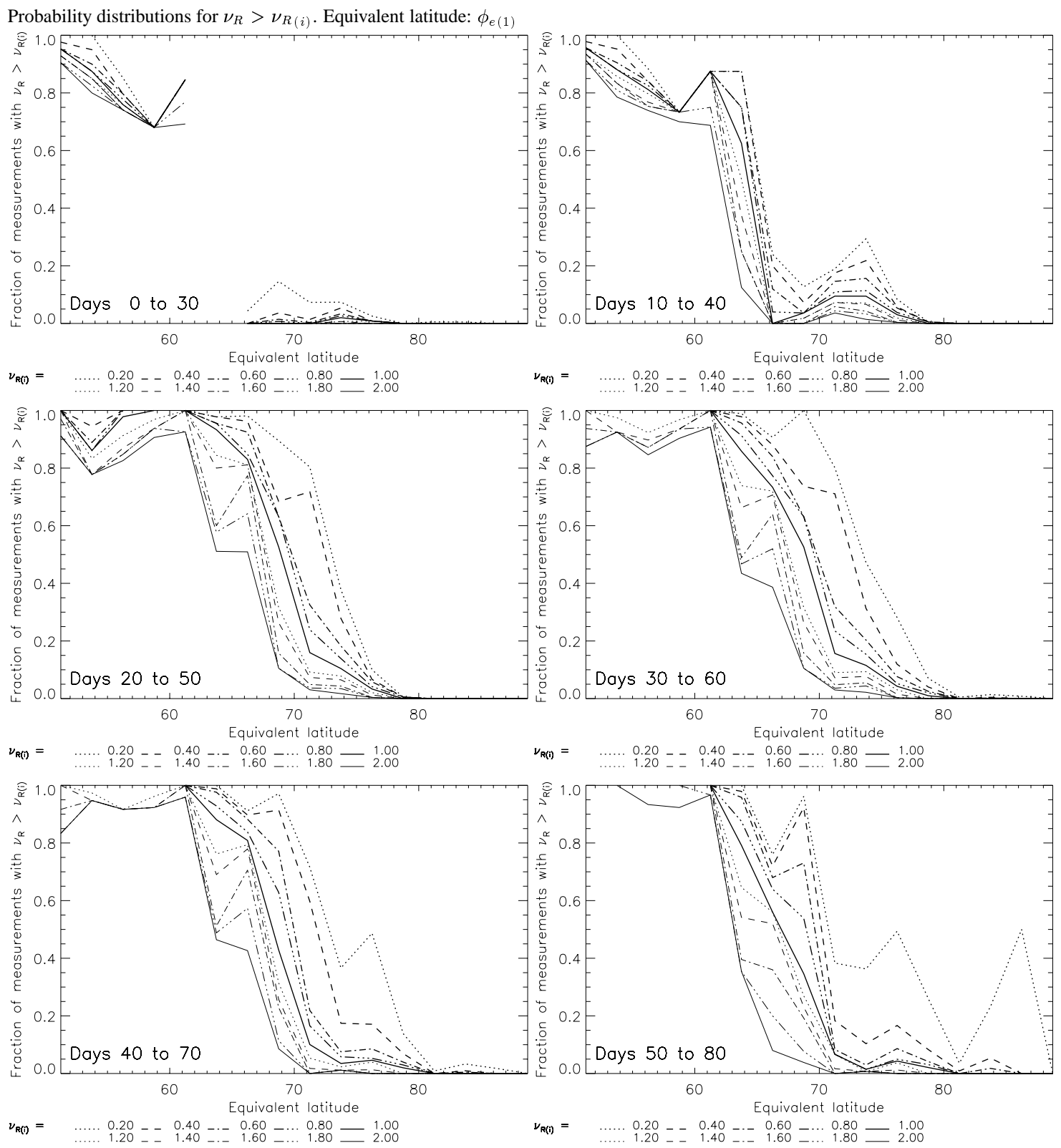

Fig. 4. As Fig. 2, but for $\phi_{e(1)}$.

line shows an idealised PDF with mean zero and Gaussian error of $2.7^{\circ}$ (the value estimated above). The points correspond to the estimated PDFs, as Fig. 2 but with the best estimate for the centre point of each PDF (the true equivalent latitude, $\left.\phi_{e(t)}\right)$ removed. The calculation of the "best estimate of $\phi_{e(t)}$ " is actually discussed later in Sect. 4 . These values of $\phi_{e(t)}$ are used here just for clarity: to demonstrate that the assumption of Gaussian error and the estimated width are appropriate for the vortex edge data. Figure 3a shows points from all the PDFs, while Fig. 3b shows points only for $v_{R(i)} \geq 0.4$ and excludes bins with less than 50 measurements. The points in Fig. $3 b$ fall mainly on a compact curve. Significant deviations from this curve occur only in Fig. 3a, i.e. where the number of measurements are low, or for low $v_{R(i)}$. The former may be regarded as noise through bad statistics.

This estimate is of course representative only of the vortex edge region. Equivalent latitude error would be expected to be very much larger away from the edge region. However, these relative errors are due to differences in the underlying dynamics, which in turn lead to very different requirements on the equivalent latitude tool in the edge, surf-zone and vortex core. The target of this paper is the detection of transport of tracer across a strong vortex edge. For detecting crossvortex transport, or in general the position of an air parcel as being within, poleward or equatorward of the vortex edge, only the error in vortex edge equivalent latitude is important. 
3.2 Improvement in equivalent latitude through PV tracer transport

All analysis thus far has made use of $\phi_{e(5)}$ as the equivalent latitude estimate. That is, equivalent latitude for each measurement point was calculated from a model tracer initialised with PV 4-5 days earlier. Figure 4 shows PDFs as in Fig. 2, but for $\phi_{e(1)}$ instead of $\phi_{e(5)}$. Figure 5 shows PDFs for $\left(\phi_{e(4)}+\phi_{e(5)}\right) / 2$.

The PDFs for $\phi_{e(1)}$ are distinctly noisier than those for $\phi_{e(5)}$, with much less tendency towards the Gaussian form. Without a consistent shape for the PDFs, it is rather difficult to define a width for them. On the other hand, if the extreme errors are ignored, it is not clear that the widths of the individual PDFs differ substantially from those for $\phi_{e(5)}$. The PDFs for $\left(\phi_{e(4)}+\phi_{e(5)}\right) / 2$ are also noticeably noisier than those for $\phi_{e(5)}$, but again the widths are similar.

$\phi_{e(5)}$, then, does offer a significant advantage over even $\left(\phi_{e(4)}+\phi_{e(5)}\right) / 2$. Passive tracer transport of PV has improved the diagnosis of equivalent latitude. The consistent Gaussian shapes of the PDFs for $\phi_{e(5)}$ allows much more confidence in the use of $\phi_{e(5)}$ for interpreting this data. Iterative tracer transport satisfies the error accumulation condition of the Central Limit Theorem. That said, it is not clear that the error distribution of $\phi_{e(5)}$ is any tighter than that of $\phi_{e(1)}$. This may be a limitation of the chosen SLIMCAT grid resolution. However, $\phi_{e(5)}$ is still clearly the best coordinate for the vortex edge analysis presented here.

\subsection{Aerosol sedimentation}

Aerosol microphysics could reduce the correlation between the lidar tracer and observed gas tracers. The correlations between ozone and aerosol surface mixing ratio observed by Borrmann et al. (1995) show significant variability between August and December 1991, probably due to early microphysical evolution of the Pinatubo aerosol. From December 1991 until early March 1992, however, the correlation is positive, linear and compact. The scatter is within measurement error.

Aerosol sedimentation could potentially affect the early 1992 lidar data analysed here. Its importance would depend on the ratio of two timescales: that for crossing of equivalent latitude contours through sedimentation $\left(\tau_{s e d}\right)$, and that for the removal of equivalent latitude anomalies through mixing $\left(\tau_{m i x}\right) . \tau_{\text {sed }}$ would be linear in sedimentation velocity, which varies strongly with altitude. For the same size distribution, the mean sedimentation velocity for $440-475 \mathrm{~K}$ is slower by a factor of 1.5 than for $475-520 \mathrm{~K}$ (using typical size distributions observed by Deshler et al. (1993) and Deshler (1994)). PDFs were calculated for data in the 440-475 K potential temperature interval. These show no difference in width from those shown above for $475-520 \mathrm{~K}$. This suggests that sedimentation may be unimportant for this analysis, but it certainly cannot be ruled out.

\section{Best estimate of the true equivalent latitude profile of the observed lidar tracer}

Having characterized the error in $\phi_{e}$ with respect to the lidar tracer, it is possible to suggest methods for filtering this error out, in order to obtain a clear precise picture of the distribution of the observed tracer in true equivalent latitude $\left(\phi_{e(t)}\right)$. Once errors in equivalent latitude are removed, the possibilities for detected true irreversible transport across barriers such as the vortex edge are much improved. In this section, two methods of filtering the equivalent latitude error out are suggested. Both methods are based on the PDFs investigated above. The base equivalent latitude tracer used here is $\phi_{e(5)}$, because of its smoother, approximately Gaussian error statistics (see Sect. 3.2).

\subsection{Method descriptions}

\subsubsection{Area-summation method}

Equivalent latitude is calculated directly from the area enclosed by a tracer contour on an isentropic surface. Nakamura (1996) showed that two dimensional transport can be written in a contour area coordinate as a diffusion. Hence, rather than trying to filter out errors in equivalent latitude directly, an alternative is to first attempt a best estimate of the area enclosed by a tracer contour. The method suggested here is called the "area-summation" method.

$A_{i}$, the isentropic area enclosed by the contour $v_{R}=v_{R(i)}$ is here estimated as follows. First, the isentrope is divided into segments of known area. For each segment, the fraction $f$ of measurements with $v_{R}>v_{R(i)}$ is found. $f$ is taken as an estimate of the area fraction of the chosen segment for which $v_{R}>v_{R(i)}$. The validity of this assumption depends on the number and distribution of observations, and on the shape of the segment. If there are many observations spread evenly, the shape of the segment is unimportant. However, if the segment is sampled sparsely, then it is best for the segment boundaries to approximate to contours of true equivalent latitude - that is, the segment should approximate to a true equivalent latitude band. This is because contours of true equivalent latitude will, by definition, follow contours of $v_{R}$ precisely. Therefore, the part of an equivalent latitude "band" which is sampled is likely to be representative of the whole band. In contrast, if for example a latitude (as opposed to equivalent latitude) band was used, it is far more likely that the sparse observations would be unrepresentative of the whole band, since it is common for vortex centre, vortex edge and midlatitude air to fall on the same latitude line. With this assumption, the total area $A_{i}$ enclosed by the contour $v_{R}=v_{R(i)}$ is easily estimated by summing over all segments:

$$
A_{i}=\sum_{k} A_{k} \cdot \frac{n_{k i}}{n_{t o t, k}}
$$



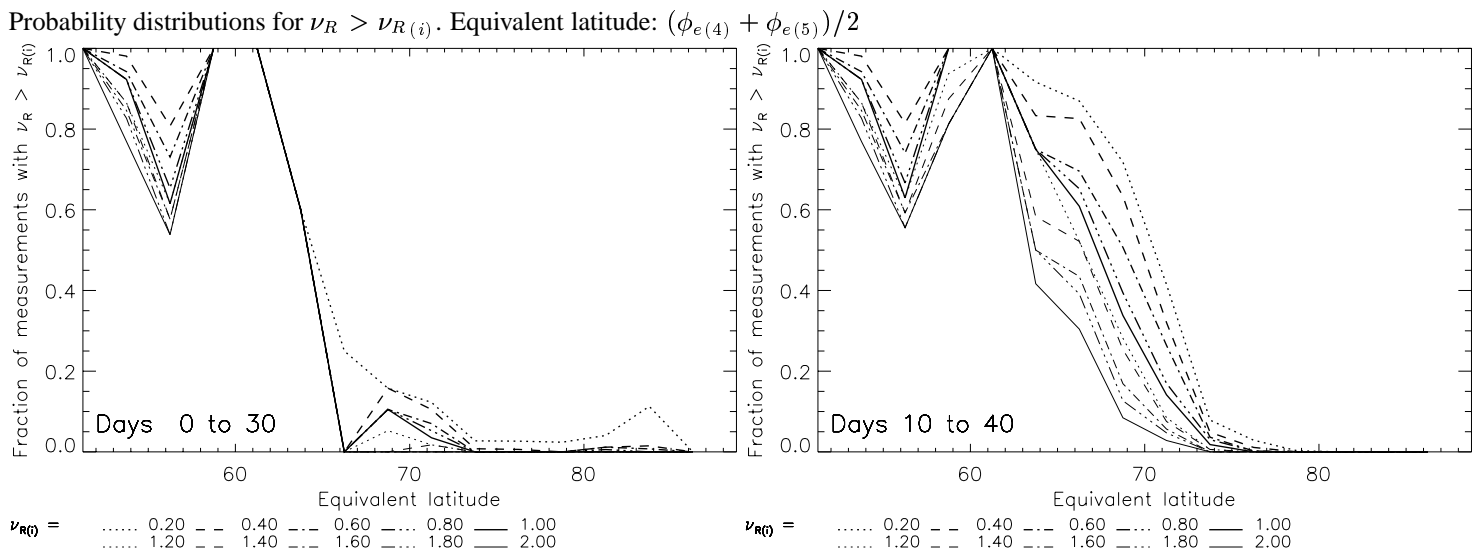

$\nu_{\mathrm{R}(i)}=\quad \ldots . .0 .20--0.40--0.60-\cdots 0.80-1.00$
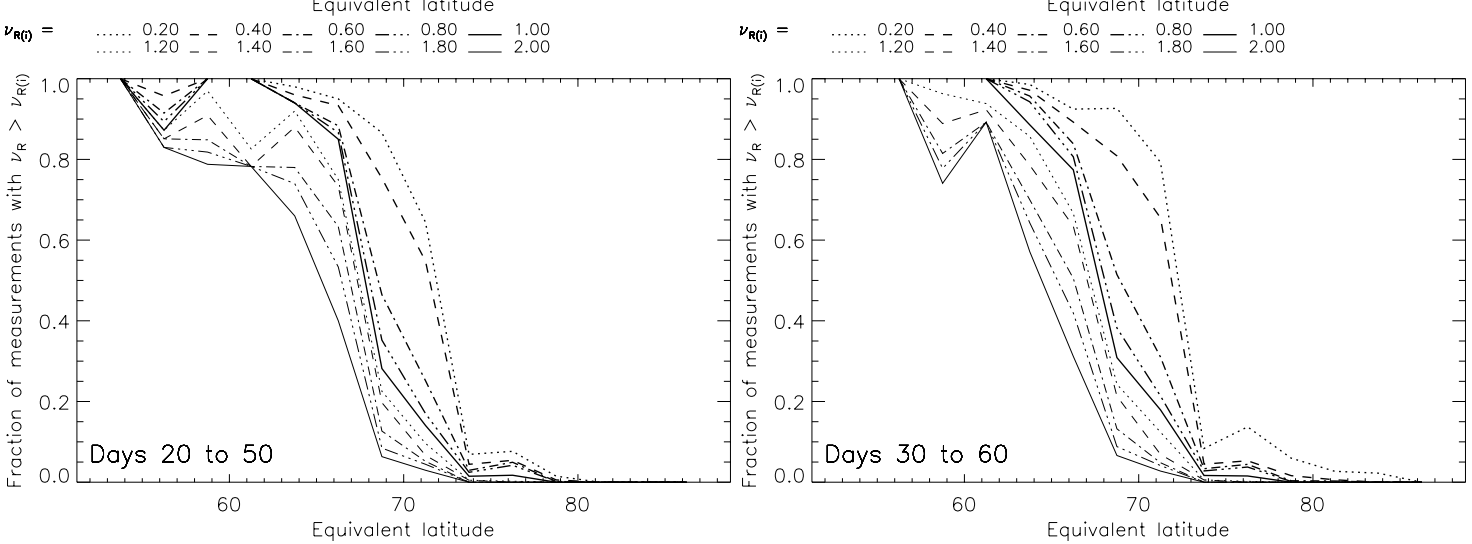

$\nu_{\mathrm{R}(i)}=\quad \ldots . .0 .20--0.40-.-0.60-\ldots 0.80=1.00$

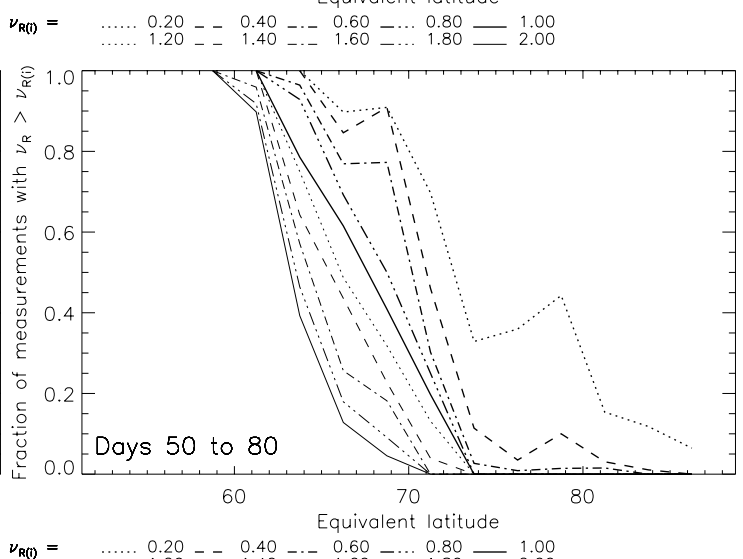

$\nu_{\mathrm{R}(\mathrm{i})}$

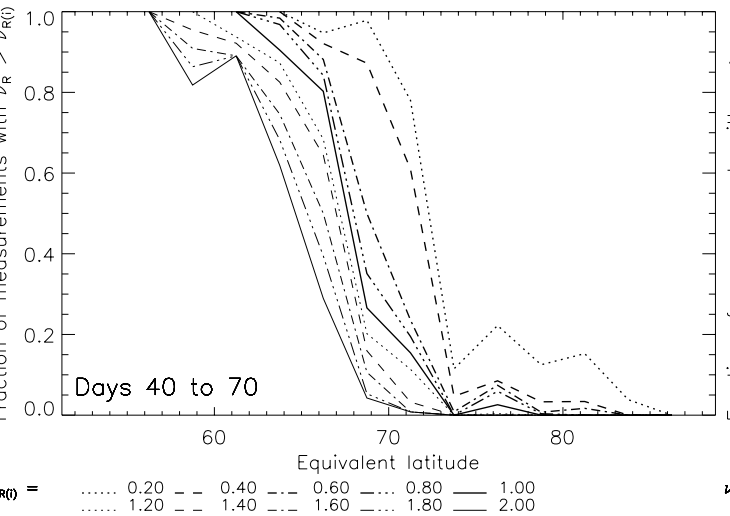

$\nu_{\mathrm{R}(i)}=$

Fig. 5. As Fig. 2, but for $\left(\phi_{e(4)}+\phi_{e(5)}\right) / 2$.

where the sum is over $\phi_{e}$ bins (or bands). $A_{k}$ is the area of the $k$ th $\phi_{e}$ bin. $\frac{\bar{n}_{k i}}{\bar{n}_{t o t, k}}$ is the fraction of measurements in $\phi_{e}$ bin $k$ for which $v_{R}>v_{R(i)}$. That is, $\frac{\bar{n}_{k i}}{\bar{n}_{t o t, k}}$ is just a PDF, like those studied in the earlier sections on error statistics.

The accuracy of $A_{i}$ depends only on the quality of the PDF estimate. There are no other assumptions, so if the PDF is accurate, then $A_{i}$ will be correct. The PDF quality depends on how well the observations sample the atmosphere. The more accurate the $\phi_{e}$ coordinate, the fewer observations are required. For sufficiently good observational sampling, $A_{i}$ will be only weakly dependent on the accuracy of $\phi_{e}$; whereas if there are few measurements then $\phi_{e}$ error is more important. Where there are obvious errors in the PDFs, these can be removed before $A_{i}$ is calculated. For Fig. 2, this means the downward spikes at $\phi_{e}<60$. Where large parts of the PDFs are unreliable (days $0-30$ and $10-40$ in Fig. 2), the area-summation method is not appropriate.

\subsubsection{Curve-fitting method}

The second, curve-fitting method, assumes that $\phi_{e}$ has Gaussian error statistics, and that the error is constant over the period studied. The appropriate curve is fitted to each PDF, 
exactly as in Sect. 3.1, except this time with the mean as the single variable parameter. The curve widths were fixed, using the value estimated in Sect. 3.1. This allows curve fits to be made where only part of the PDF is reliable (days 0-30 and 10-40 in Fig. 2), which is the major advantage of this method. The assumption here is that the Gaussian error in $\phi_{e}$ is constant, since the PDF curve width is determined by the Gaussian error in $\phi_{e}$. The weighting was the same as in Sect. 3.1. This method only works well for $\phi_{e(5)}$, since equivalent latitude from PV tracers advected for shorter times do not have Gaussian error statistics (section 3.2)

\subsubsection{Discussion}

The area-summation method makes the fewest assumptions, and so is preferred where the obvious PDF errors are not extensive. An alternative would be to take mean $\phi_{e}$ for narrow $v_{R}$ bins. However, the use of the mean makes specific assumptions about the statistics of the error in $\phi_{e}$, which may well not be precisely correct, introducing systematic error into the results. The above area-summation method makes rather weaker assumptions about the form of the error and uses much more of the data. Both area-summation and curvefitting methods are based directly on the PDFs, which have been investigated in detail, so the reliability of the results will be better understood.

We are interested in time-resolved tracer transport. Thus, for each estimate of $\phi_{e(t)},\left(\phi_{e(t, i)}\right.$, say) a corresponding time coordinate value must be assigned - a day of the year $\left(t_{i}\right)$. Each estimate of $\phi_{e(t)}$ is based on multiple observations taken at irregular intervals. However, each such $\phi_{e(t)}$ estimate is controlled by those observations at equivalent latitudes near the PDF centre. A sensible value for $t_{i}$ then, is a weighted mean of the observation times, with the weights given by a Gaussian of width $2.7^{\circ}$ centred on $\phi_{e(t)}$. The results presented in Sect. 4.3 use this approach to present $\phi_{e(t)}$ as a function of time. This weighted mean time was found to give much smoother results than a simple unweighted mean of the observation times.

\subsection{Method performance}

The two methods were applied to the following time bins, which show reasonable Gaussian statistics: days 20-50, 30$60,40-70$ and 50-80. The difference between the two estimates is summarised as $-0.04 \pm 0.25^{\circ}$. This standard deviation of $\pm 0.25^{\circ}$ gives a rough estimate for the random error in the calculated true equivalent latitude $\phi_{e(t)}$. It was calculated using a maximum likelihood correction to the standard deviation that assumes 6 statistically independent comparisons. The value of 6 independent comparisons was estimated very roughly by examining how the PDFs overlap in time and equivalent latitude bins. This is sufficiently precise for the interpretation of the results presented below. The curve-fitting method shows results which are noisier than the area-summation method; therefore, for sufficiently reliable PDFs, the area-summation method is used as the best estimate of $\phi_{e(t)}$.

For days $0-30$ and $10-40$, the PDFs are too poorly sampled for the area-summation method to work. It was found above that the parts of PDFs based on less than 50 measurements tend to be unreliable. For days $0-30$ and 10-40, the curve-fitting method only was applied. These curve fits use only part of the PDF - those parts where there are sufficient measurements for the PDF to be reliable. This is the intention of the weighting scheme used. For example, the fit for $v_{R(i)}=0.2$, days $0-30$ is controlled by the point at $\phi_{e}=71.25$. This point on the PDF was based on a large number of measurements (about 200) and so the weighting assigned to it was much higher than for the points for $\phi_{e}<71.25$, to which rather fewer measurements contributed (see top panel of Fig. 2). The spike in $v_{R}$ at $\phi_{e}>78$ for days $0-30$ is very likely due to PSC contamination, and was discarded from the analysis. This leaves the point at $\phi_{e}=71.25$ as the only one with both a useful number of measurements and a non-zero PDF value (0.03). The performance of curve fits that use only a single point from this part of the PDF was checked as follows. The main reason for this check is that the assumption of Gaussian statistics is not precisely correct. This check was done using the reliable, complete PDFs, from day bins 20-50 onwards, and for $\nu_{R(i)}>0.2$. For these data the $\phi_{e(t)}$ estimate was subtracted from each PDF, as in Fig. 3. Then, a bias and random error was calculated from the distribution of PDF points around the $2.7^{\circ} \mathrm{Gaussian}$ in the neighbourhood of the probability value 0.03 ( 0.03 is the PDF value for the point controlling the curve fit for days $\left.0-30, v_{R(i)}=0.2\right)$. This yields a bias and a random error for the estimate of $\phi_{e(t)}$ using a partial curve fit for days $0-30$. For the probability interval $0.02-0.1$, the curve fit error is fairly uniform: a bias of $0.6^{\circ}$ and a maximum likelihood random error of $1.1^{\circ}$. For days $10-40$, and $v_{R(i)}=2.0$ a bias of $0.4^{\circ}$ and random error of $1.0^{\circ}$ was found. For days $10-40$, and $v_{R(i)}<1.8$ the bias becomes zero. A bias arises in the extremes of the PDFs because the fitted Gaussian-based curve is not precisely the right form. The estimated bias can be used to correct $\phi_{e(t)}$.

\subsection{Results}

Figure 6 shows the resulting best estimates of $\phi_{e(t)}$, as a function of $v_{R}$ and time, with $1-\sigma$ error bars. For time bins after days 20-50 (the rightmost four points on each line) the area-summation method is used; for days $0-30$ and 10-40 the results are from curve-fitting with bias corrections. Some points are not plotted where the error bars overlap (near day 32 ). The ordinate is linear in contour enclosure area: Nakamura (1996) showed that isentropic tracer transport can be modelled by simple diffusion in an area coordinate. Abscissa values are Gaussian-weighted means, as described above.

Vertical gradients within the $475-520 \mathrm{~K}$ potential temperature interval are small. This was determined by producing 
similar plots for the $475-495 \mathrm{~K}$ and $495-520 \mathrm{~K}$ sub-intervals. The results differed by less than $1^{\circ}$, except for the days $50-80$ time bin which has rather few observations.

The results (Fig. 6) show a steep, mostly stationary vortex edge gradient, ending at $70-72^{\circ}$. This is consistent with the vortex edge location in February 1992 UKMO analyses. There may be a small poleward shift of about $1^{\circ}$ per month, also seen in the UKMO PV gradient. The $v_{R}=0.2$ contour (either inside the vortex or at the poleward extent of the vortex edge) shows very clear poleward motion, probably indicating irreversible poleward aerosol transport. A substantial rise in the frequency of observations with $v_{R}>0.2$ at $\phi_{e}>70^{\circ}$ after day 40 is visible independently in the stations at Andoya, Ny-Ålesund and Thule. Thus, there appears to have been significant transport across the vortex edge during late January. This is not conclusive, since it is not possible to determine the precise extent of the vortex edge itself. However, the PDFs in Fig. 2 give some support. Specifically, the PDF for $v_{R(i)}<0.2$ and days 50-80 has a very different shape from that for earlier times. for $\phi_{e}>71^{\circ}$, the PDF is stretched poleward as far as $\phi_{e}=90^{\circ}$. This indicates a different form for the $\phi_{e}$ error statistics, suggesting that different dynamics are in play for $v_{R(i)}=0.2$ and days 50-80. Secondly, Good and Pyle (2004) demonstrate transport of volcanic aerosol to the very highest equivalent latitudes (as opposed to simply entering the vortex core) starting just below $475 \mathrm{~K}$. Thus it seems likely that some transport into the vortex core occurred above $475 \mathrm{~K}$. This irreversible transport may be associated with the vortex disturbance during this period, which was demonstrated in contour advection simulations and airborne lidar data by Plumb et al. (1994). The time lag between the disturbance reported by Plumb et al. (1994) and the irreversible transport indicated in Fig. 6 is expected. Irreversible transport would have occurred only if and when the vortex intrusion had been stretched by advection sufficiently for turbulent mixing to act efficiently. The process of mixdown, and mix-down times are discussed by Thuburn and Tan (1997) and Good et al. (2003).

The equivalent latitude width of the steep vortex edge lidar tracer gradient may be estimated. In Fig. 6, the gradient for $0.6 \leq v_{R(t)} \leq 2$ is $0.3 \pm 0.02$ per degree. The scatter plot of $v_{R}$ versus $\phi_{e}$ (Fig. 1) shows that the steep gradient region is bounded equator-ward by $v_{R} \approx 3-4$ and poleward by $v_{R} \approx 0$. This suggests a total width for the steep vortex edge lidar tracer gradient of about $11 \pm 2^{\circ}$. There is no significant difference between this and the width of the vortex edge gradient in UKMO analysed PV. This is not necessarily what would be expected, because observed vortex edge tracer gradients are generally narrow compared to those for PV. The vortex-edge PV gradient is broadened by radiative relaxation, but the same cannot occur for constituent tracers such as aerosol (e.g. Haynes and Anglade, 1997). The vertical resolution of the observed data used here is relatively high, at $200 \mathrm{~m}$. A broadening effect due to aerosol sedimentation cannot be ruled out. Most likely, the broad width of

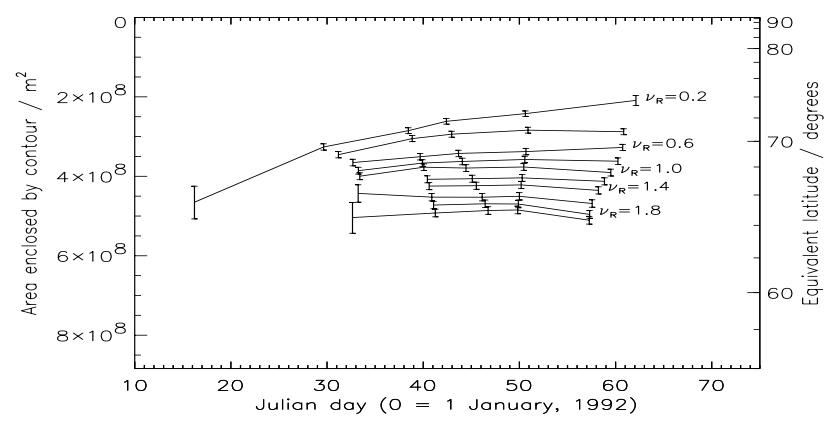

Fig. 6. Best estimates of true equivalent latitude for selected $v_{R}$ values. Before day 35: curve-fit method with bias corrections; after day 35: area-summation method.

the vortex-edge gradient in $v_{R}$ observed here is due to mixing across the disturbed vortex edge that appears to have occurred during late January.

\section{Conclusions}

In conclusion an approach has been developed to extract very precise and reliable information from sporadic, inhomogeneously distributed tracer data. A very large number of relatively high resolution observations were analysed alongside tracer-based equivalent latitude fields.

The broad approach was to understand equivalent latitude errors, and use this knowledge to guide assimilation of the measurements, particularly where there are few observations. The overall approach is in practice an optimisation procedure, since an improved knowledge of the distribution of the observed tracer feeds back with help in understanding the errors in equivalent latitude. This approach may be useful in filling in gaps in satellite observation grids more accurately. The analysis was based around probability distribution estimates, for $v_{R}>v_{R(i)}$ as a function of equivalent latitude. Such PDFs have been used in previous studies (e.g. Sparling, 2000), but expressed as a function of latitude rather than equivalent latitude, imposing restrictions on their usefulness. The method works for these data in the critically important vortex edge region: where measurement error is small compared to equivalent latitude error and the observed tracer can be assumed to be locally monotonic in true equivalent latitude.

A new method was demonstrated for estimating the random error in diagnosed equivalent latitude against observed data. For the equivalent latitude field used here a Gaussian error of $2.6 \pm 0.25^{\circ}$ was found. This is the first time that equivalent latitude error has been quantified in this way. The results have implications for the use of equivalent latitude to interpret observations. For example, for this selection of equivalent latitude and observations, the classification of a single measurement as "inside the vortex centre" has a better then 
$99 \%$ certainty only if $\phi_{e}$ is more than $8^{\circ}$ inside the diagnosed vortex edge poleward boundary. This result depends on the model configuration, forcing winds and specifics of the observed tracer, although experiments with higher resolution model transport have shown no significant reduction in error. Allen and Nakamura (2003) demonstrate a new method for calculating a PV-like tracer which may be smoother at high latitude, and potentially lead to lower equivalent latitude errors within the vortex edge. The estimated $\phi_{e}$ error may have been increased by aerosol sedimentation. The lack of vertical variation in $\phi_{e}$ error may indicate that sedimentation has not caused a significant overestimate, but it is difficult to rule out.

This method was applied to demonstrate fairly clear benefits of passive Eulerian tracer transport of PV in modifying the error in equivalent latitude fields. The effect found here was to drive the error statistics towards a Gaussian distribution, although there was not a significant change in the mean error. Equivalent latitude based on a PV-initialised tracer will be improved if the local mixing is faster than the introduction of error by numerical model transport. The mechanism is analogous to that used by Plumb and Ko (1992) to explain the tightening of tracer-tracer interrelationships. This is different from the effect of high resolution Lagrangian transport with no imposed spatial averaging, which reduce the spatial scales of equivalent latitude errors - without reducing the statistical distribution of equivalent latitude errors, balanced by the steady addition of error through model transport. Similarly, the equivalent latitude error estimate in this paper differs from the filament spatial position error estimate made by Flentje et al. (2000) for contour advection results.

Some new methods were also demonstrated for estimating precisely the true equivalent latitude profile of observed vortex edge tracer data, within a fixed potential temperature interval. Such precise estimates are essential for detecting true irreversible transport across barriers such as the vortex edge. Simple plots of observed tracer against diagnosed equivalent latitude (such as Fig. 1) are inadequate for this purpose, since any apparent transport may in fact be an artifact of error in the equivalent latitude coordinate. In the vortex edge region selected, equivalent latitude error is much more important than measurement random error. Understanding the $\phi_{e}$ error statistics allowed the precision, accuracy and domain of the results to be improved and their precision estimated. In particular, the significance of good or poor sampling densities can be seen, and where sampling is poor, the appropriate methods for estimating the true equivalent latitude can be applied. Conventional binning and tracer averaging would have obscured the detailed picture significantly.

A small amount of poleward transport across the vortex edge in the $475-520 \mathrm{~K}$ interval was detected. This appears related to the strong vortex disturbance observed in late January by Plumb et al. (1994). Good and Pyle (2004) show that poleward transport increased below $475 \mathrm{~K}$ and was negligible above $520 \mathrm{~K}$, so the interval $475-520 \mathrm{~K}$ is a kind of transition region. The equivalent latitude width of the vortex edge steep tracer gradient shows no difference from that of the PV gradient, probably due to mixing across the disturbed late-January vortex edge. This is not obvious, since radiative relaxation may broaden the PV gradient compared to constituent tracer gradients (e.g. Haynes and Anglade, 1997). A contribution from sedimentation could play some part in either of these results.

Edited by: K. Hamilton

\section{References}

Allen, D. R. and Nakamura, N.: Tracer equivalent latitude: A diagnostic tool for isentropic transport studies, J. Atmos. Sci., 60, 287-304, 2003.

Beyerle, G. and Neuber, R.: The stratospheric aerosol content above Spitsbergen during winter 1991/92, Geophys. Res. Lett., 21, 1291-1294, 1994.

Bohren, C. and Huffman, D.: Scattering and Absorption of Light by Small Particles, Wiley, New York, 1981.

Borrmann, S., Dye, J. E., Baumgardner, D., Proffitt, M. H., Margitan, J. J., Wilson, J. C., Jonsson, H. H., Brock, C. A., Loewenstein, M., Podolske, J. R., and Ferry, G. V.: Aerosols as dynamical tracers in the lower stratosphere - ozone versus aerosol correlation after the Mount-Pinatubo eruption, J. Geophys. Res.Atmos., 100, 11 147-11 156, 1995.

Brock, C. A., Jonsson, H. H., Wilson, J. C., Dye, J. E., Baumgardner, D., Borrmann, S., Pitts, M. C., Osborn, M. T., Decoursey, R. J., and Woods, D. C.: Relationships between optical extinction, backscatter and aerosol surface and volume in the stratosphere following the eruption of Mt-Pinatubo, Geophys. Res. Lett., 20, 2555-2558, 1993.

Browell, E. V., Butler, C. F., Ismail, S., Robinette, P. A., Carter, A. F., Higdon, N. S., Toon, O. B., Schoeberl, M. R., and Tuck, A. F.: Airborne lidar observations in the wintertime Arctic stratosphere - polar stratospheric clouds, Geophys. Res. Lett., 17, 385388, 1990.

Butchart, N. and Remsberg, E. E.: The area of the stratospheric polar vortex as a diagnostic for tracer transport on an isentropic surface, J. Atmos. Sci., 43, 1319-1339, 1986.

Chazette, P., David, C., Lefrere, J., Godin, S., Pelon, J., and Megie, G.: Comparative lidar study of the optical, geometrical, and dynamical properties of stratospheric post-volcanic aerosols, following the eruptions of El-Chichon and Mount-Pinatubo, J. Geophys. Res.-Atmos., 100, 23 195-23 207, 1995.

Chipperfield, M. P.: Multiannual simulations with a threedimensional chemical transport model, J. Geophys. Res., 104, 1781-1805, 1999.

Chipperfield, M. P. and Jones, R. L.: Relative influences of atmospheric chemistry and transport on Arctic ozone trends, Nature, 400, 551-554, 1999.

Deshler, T.: In-situ measurements of Pinatubo aerosol over Kiruna on 4 days between 18 January and 13 February 1992, Geophys. Res. Lett., 21, 1323-1326, 1994.

Deshler, T., Johnson, B. J., and Rozier, W. R.: Balloonborne measurements of Pinatubo aerosol during 1991 and 1992 at 41- 
degrees-N - vertical profiles, size distribution, and volatility, Geophys. Res. Lett., 20, 1435-1438, 1993.

Digirolamo, P., Cacciani, M., Disarra, A., Fiocco, G., and Fua, D.: Lidar observations of the Pinatubo aerosol layer at Thule, Greenland, Geophys. Res. Lett., 21, 1295-1298, 1994.

Dragani, R., Redaelli, G., Visconti, G., Mariotti, A., Rudakov, V., MacKenzie, A. R., and Stefanutti, L.: High-resolution stratospheric tracer fields reconstructed with Lagrangian techniques: A comparative analysis of predictive skill, J. Atmos. Sci., 59, 1943-1958, 2002.

Esler, J. G., Polvani, L. M., and Plumb, R. A.: On the mix-down times of dynamically active potential vorticity filaments, Geophys. Res. Lett., 26, 2953-2956, 1999.

Evans, M. J., Shallcross, D. E., Law, K. S., Wild, J. O. F., Simmonds, P. G., Spain, T. G., Berrisford, P., Methven, J., Lewis, A. C., McQuaid, J. B., Pilling, M. J., Bandy, B. J., Penkett, S. A., and Pyle, J. A.: Evaluation of a Lagrangian box model using field measurements from EASE (Eastern Atlantic Summer Experiment) 1996, Atmos. Environ., 34, 3843-3863, 2000.

Fairlie, T. D., Pierce, R. B., Grose, W. L., Lingenfelser, G., Loewenstein, M., and Podolske, J. R.: Lagrangian forecasting during ASHOE/MAESA: Analysis of predictive skill for analyzed and reverse-domain-filled potential vorticity, J. Geophys. Res.Atmos., 102, 13 169-13 182, 1997.

Farman, J. C., Oneill, A., and Swinbank, R.: The dynamics of the arctic polar vortex during the EASOE campaign, Geophys. Res. Lett., 21, 1195-1198, 1994.

Flentje, H., Renger, W., and Wirth, M.: Validation of Contour Advection simulations with airborne lidar measurements of filaments during the Second European Stratospheric Arctic and Midlatitude Experiment (SESAME), J. Geophys. Res.-Atmos., 105, 15 417-15 437, 2000.

G. Brasseur, J. O. and Tyndall, G.: Atmospheric Chemistry and Global Change, Oxford University Press, New York, 1999.

Good, P. and Pyle, J.: Refinements in the use of equivalent latitude for assimilating sporadic inhomogeneous stratospheric tracer observations, 2: Precise altitude-resolved information about transport of Pinatubo aerosol to very high latitude, Atmos. Chem. Phys. Discuss., Vol. 4, 67-693, 29-1-200420042, 2004.

Good, P., Giannakopoulos, C., O'Connor, F. M., Arnold, S. R., de Reus, M., and Schlager, H.: Constraining tropospheric mixing timescales using airborne observations and numerical models, Atmos. Chem. Phys., 3, 1023-1035, 2003,

SRef-ID: 1680-7324/acp/2003-3-1023.

Hanson, D. R., Ravishankara, A. R., and Solomon, S.: Heterogeneous reactions in sulfuric-acid aerosols - a framework for model-calculations, J. Geophys. Res.-Atmos., 99, 3615-3629, 1994.

Haynes, P. and Anglade, J.: The vertical-scale cascade in atmospheric tracers due to largescale differential advection, J. Atmos. Sci., 54, 1121-1136, 1997.

Jager, H., Deshler, T., and Hofmann, D. J.: Midlatitude lidar backscatter conversions based on balloonborne aerosol measurements, Geophys. Res. Lett., 22, 1729-1732, 1995.

Manney, G. L., Bird, J. C., Donovan, D. P., Duck, T. J., Whiteway, J. A., Pal, S. R., and Carswell, A. I.: Modeling ozone laminae in ground-based Arctic wintertime observations using trajectory calculations and satellite data, J. Geophys. Res.-Atmos., 103, 5797-5814, 1998.
Massie, S. T., Dye, J. E., Baumgardner, D., Randel, W. J., Wu, F., Tie, X. X., Pan, L. W., Figarol, F., Brasseur, G. P., Santee, M. L., Read, W. G., Grainger, R. G., Lambert, A., Mergenthaler, J. L., and Tabazadeh, A.: Simultaneous observations of Polar Stratospheric Clouds and HNO3 over Scandinavia in January, 1992, Geophys. Res. Lett., 24, 595-598, 1997.

Methven, J. and Hoskins, B.: The advection of high-resolution tracers by low-resolution winds, J. Atmos. Sci., 56, 3262-3285, 1999.

Methven, J., Evans, M., Simmonds, P., and Spain, G.: Estimating relationships between air mass origin and chemical composition, J. Geophys. Res.-Atmos., 106, 5005-5019, 2001.

Methven, J., Arnold, S., O'Connor, F., Barjat, H., Dewey, K., Kent, J., and Brough, N.: Estimating photochemically produced ozone throughout a domain using flight data and a Lagrangian model, J. Geophys. Res., accepted, 2003.

Millard, G. A., Lee, A. M., and Pyle, J. A.: A model study of the connection between polar and midlatitude ozone loss in the Northern Hemisphere lower stratosphere, J. Geophys. Res.Atmos., 108, art. no. 8323, 2002.

Nakamura, N.: Two-dimensional mixing, edge formation, and permeability diagnosed in an area coordinate, J. Atmos. Sci., 53, 1524-1537, 1996.

O'Neill, A., Grose, W. L., Pope, V. D., MacLean, H., and Swinbank, R.: Evolution of the stratosphere during northern winter 1991/92 as diagnosed from UK-Meteorological-Office analyses, J. Atmos. Sci., 51, 2800-2817, 1994.

Plumb, R. A. and Ko, M. K. W.: Interrelationships between mixing ratios of long lived stratospheric constituents, J. Geophys. Res.Atmos., 97, 10 145-10 156, 1992.

Plumb, R. A., Waugh, D. W., Atkinson, R. J., Newman, P. A., Lait, L. R., Schoeberl, M. R., Browell, E. V., Simmons, A. J., and Loewenstein, M.: Intrusions into the lower stratospheric arctic vortex during the winter of 1991-1992, J. Geophys. Res.-Atmos., 99, 1089-1105, 1994.

Plumb, R. A., Waugh, D. W., and Chipperfield, M. P.: The effects of mixing on traces relationships in the polar vortices, J. Geophys. Res.-Atmos., 105, 10 047-10 062, 2000.

Prather, M. J.: Numerical advection by conservation of 2nd-order moments, J. Geophys. Res., 91, 6671-6681, 1986.

Russell, J. M., Gordley, L. L., Park, J. H., Drayson, S. R., Hesketh, W. D., Cicerone, R. J., Tuck, A. F., Frederick, J. E., Harries, J. E., and Crutzen, P. J.: The halogen occultation experiment, J. Geophys. Res., 98, 10777-10 797, 1993.

Russell, P. B., Livingston, J. M., Pueschel, R. F., Bauman, J. J., Pollack, J. B., Brooks, S. L., Hamill, P., Thomason, L. W., Stowe, L. L., Deshler, T., Dutton, E. G., and Bergstrom, R. W.: Global to microscale evolution of the Pinatubo volcanic aerosol derived from diverse measurements and analyses, J. Geophys. Res.-Atmos., 101, 18 745-18 763, 1996.

Schafer, H. J., Scheuch, P., Langer, M., Fricke, K. H., Vonzahn, U., and Knudsen, B. M.: Lidar observations of polar stratospheric clouds at Andoya, Norway, in January 1992, Geophys. Res. Lett., 21, 1307-1310, 1994.

Sparling, L. C.: Statistical perspectives on stratospheric transport, Rev. Geophys., 38, 417-436, 2000.

Steele, H. M. and Hamill, P.: Effects of temperature and humidity on the growth and opticalproperties of sulfuric acid-water droplets in the stratosphere, J. Aerosol. Sci., 12, 517-528, 1981. 
Stein, B., Delguasta, M., Kolenda, J., Morandi, M., Rairoux, P., Stefanutti, L., Wolf, J. P., and Woste, L.: Stratospheric aerosolsize distributions from multispectral lidar measurements at Sodankyla during EASOE, Geophys. Res. Lett., 21, 1311-1314, 1994.

Sutton, R. T., MacLean, H., Swinbank, R., O’Neill, A., and Taylor, F. W.: High-resolution stratospheric tracer fields estimated from satellite-observations using Lagrangian trajectory calculations, J. Atmos. Sci., 51, 2995-3005, 1994.

Swinbank, R. and O'Neill, A.: A stratosphere troposphere data assimilation system, Monthly Weather Review, 122, 686-702, 1994.
Thuburn, J. and Tan, D. G. H.: A parameterization of mixdown time for atmospheric chemicals, J. Geophys. Res.-Atmos., 102, 13 037-13 049, 1997.

Vaughan, G., Wareing, D. P., Jones, S. B., Thomas, L., and Larsen, N.: Lidar measurements of Mt-Pinatubo aerosols at Aberystwyth from August 1991 through March 1992, Geophys. Res. Lett., 21, 1315-1318, 1994. 